\title{
Cyclin D1 harboring the T286I mutation promotes oncogenic activation in endometrial cancer
}

\author{
YUJI IKEDA $^{1}$, KATSUTOSHI ODA ${ }^{1}$, OSAMU HIRAIKE-WADA ${ }^{1}$, TAKAHIRO KOSO $^{1,2}$, \\ AKI MIYASAKA ${ }^{1}$, TOMOKO KASHIYAMA ${ }^{1}$, MICHIHIRO TANIKAWA $^{1}$, KENBUN SONE $^{1}$, \\ KAZUNORI NAGASAKA ${ }^{1}$, DAICHI MAEDA ${ }^{2}$, KEI KAWANA ${ }^{1}$, SHUNSUKE NAKAGAWA ${ }^{3}$, \\ MASASHI FUKAYAMA ${ }^{2}$, OSAMU TETSU ${ }^{4}$, TOMOYUKI FUJII ${ }^{1}$, TETSU YANO ${ }^{1}$ and SHIRO KOZUMA ${ }^{1}$ \\ Departments of ${ }^{1}$ Obstetrics and Gynecology, and ${ }^{2}$ Pathology, Faculty of Medicine, The University of Tokyo, \\ Tokyo 113-8655; ${ }^{3}$ Department of Obstetrics and Gynecology, Teikyo University, Tokyo 173-0003, Japan; \\ ${ }^{4}$ Cancer Research Institute and Comprehensive Cancer Center, University of California, San Francisco, CA 94143, USA
}

Received February 26, 2013; Accepted April 15, 2013

DOI: 10.3892/or.2013.2515

\begin{abstract}
Cyclin D1 is an important regulator of cell cycle progression. Phosphorylation of cyclin D1 at Thr286 by GSK3 $\beta$ triggers its nuclear export and cytoplasmic proteolysis via the $26 \mathrm{~S}$ proteasome. Cyclin D1 overexpression is a common event in various types of human cancers; however, reports of mutations are extremely rare. We analyzed mutations of the cyclin D1 gene, $C C N D 1$, in 88 endometrial cancer tissue specimens and detected mutations in 2 cases (2.3\%). Both were unreported mutations with substitution of threonine to isoleucine at codon 286 (T286I). These two tumors harbored coexisting mutations in $K$-ras, PIK3CA and/or PTEN and showed accumulation of cyclin D1 in the nucleus by immunohistochemistry. Furthermore, we analyzed the functions of mutant cyclin D1 (T286I) by luciferase assays, immunofluorescence, western blotting and clonogenic cell survival assays in HEK-293T cells. We found that exogenous mutant cyclin D1 (T286I) accumulated in the nuclei in HEK-293T cells, and that it inhibited the expression of $\mathrm{pRb}$. Additionally, the number of colonies was increased by introduction of mutant cyclin D1 (T286I) compared to that of wild-type cyclin D1. In conclusion, we identified an unreported CCND1 mutation (T286I) in two endometrial cancers and revealed that the mutation was functional for inducing cell proliferation in human cells.
\end{abstract}

\section{Introduction}

Endometrial cancer is the eighth leading cause of cancerrelated mortality among women (1). Approximately $80 \%$ of

Correspondence to: Dr Katsutoshi Oda, Department of Obstetrics and Gynecology, Faculty of Medicine, The University of Tokyo, 7-3-1 Hongo Bunkyo-ku, Tokyo 113-8655, Japan

E-mail: katsutoshi-tky@umin.ac.jp

Key words: cyclin D1, T286I, mutation, endometrial cancer endometrial carcinomas are endometrioid endometrial cancers (EECs) and are generally considered as estrogen-dependent tumors (2). EECs frequently possess mutations in PI3K (phosphatidylinositol 3-kinase) pathway genes including PTEN, PIK3CA, K-ras and AKTI (3-7).

Cyclin D1, encoded by the CCND1 gene, is located on chromosome 11q13 and plays a crucial role in cell cycle regulation (8). Cyclin D1 interacts with cyclin-dependentkinase 4 and 6 (CDK4/6), and the complex phosphorylates retinoblastoma protein $(\mathrm{pRb})$ to promote cell cycle progression from $\mathrm{G} 1$ to $\mathrm{S}$ phase (9-11). Cyclin D1 is activated by upstream inputs, including the Wnt- $\beta$-catenin and Ras/PI3K signaling pathways $(11,12)$. Degradation of cyclin D1 is regulated by GSK3 $\beta$, a downstream target of the PI3K pathway. The phosphorylation of cyclin D1 at Thr-286 by GSK $3 \beta$ triggers its nuclear export and cytoplasmic proteolysis via the $26 \mathrm{~S}$ proteasome (13). A polymorphism of CCND1 (G/A870), encoding cyclin D1b, was reported to influence cancer risk and prognostic outcome (14). Cyclin D1b has a different configuration at the C-terminal domain, which includes Thr286, and is constitutively localized to the nucleus $(15,16)$. Thus, this polymorphism (G/A870) is suggested to increase the risk of endometrial cancer, possibly through nuclear accumulation of cyclin D1 (17). Overexpression of cyclin D1 is a common event in various types of human cancers, including those of the breast, lung, bladder, esophagus and endometrium (13). However, mutations of $C C N D 1$ have only been reported in endometrial and esophageal cancer $(18,19)$. In the present study, we detected a novel CCND1 mutation in endometrial cancer, and analyzed whether this T286I mutation causes gain-of-function.

\section{Materials and methods}

Tumor samples and genomic DNA. Surgical samples were obtained from 88 patients with primary endometrial carcinoma who underwent resection of their tumors at the University of Tokyo Hospital. All of the patients provided informed consent for the collection and use of their samples, and the use of 
Table I. Clinicopathological background of all cases $(n=88)$.

\begin{tabular}{lc}
\hline Variables & No. of patients $(\%)$ \\
\hline Median age (range) in years & $55(28-79)$ \\
FIGO stage & \\
I & $39(44)$ \\
II & $9(10)$ \\
III & $31(35)$ \\
IV & $9(10)$ \\
Histological subtype & \\
Endometrioid adenocarcinoma & $80(91)$ \\
Grade 1 & $43(49)$ \\
Grade 2 & $28(32)$ \\
Grade 3 & $9(10)$ \\
Adenosquamous carcinoma & $3(3)$ \\
Squamous cell carcinoma & $1(1)$ \\
Clear cell carcinoma & $1(1)$ \\
Mixed type & $3(3)$ \\
Lymph metastasis & $20(23)$ \\
Lymph-vascular invasion & $39(44)$ \\
Muscle invasion (>1/3) & $53(60)$ \\
\hline
\end{tabular}

tissues for the present study was approved by the appropriate institutional ethics committees. Histopathologically, 80 out of 88 cases $(91 \%)$ were EECs (Table I). Genomic DNA was isolated from the tumor sections or lymphocyte pellets as previously described (7).

$P C R$ and sequencing. The primer sequences of exon 5 for the $C C N D 1$ gene were as follows: forward 5'-TGCTGGAGTCA AGCCTGCG-3' and reverse 5'-ACTGTCAGGGGAGCA CCTG-3'. The PCR products were sequenced using the BigDye (Applied Biosystems, Foster City, CA, USA) terminator method on an autosequencer. Mutations for PTEN (exons 1-8), $K$-Ras (exons 1 and 2), PIK3CA (exons 9 and 20) and CTNNB1 (exon 3 ) were analyzed as previously described $(3,4,7,20)$.

Immunohistochemistry (IHC). Immunohistochemical staining was performed according to standard techniques using a Ventana Benchmark XT autostainer (Ventana Medical Systems Inc., Tucson, AZ, USA). Anti-cyclin D1 rabbit monoclonal antibody (\#N161987; Dako, Carpinteria, CA, USA) was applied at a dilution of 1:100.

Cell lines and transfection. The human embryonic kidney HEK293T cell line was obtained from the American Type Culture Collection (ATCC; Manassas, VA, USA). The T286I cyclin D1 mutant vector was generated using the QuickChange Lightning Site-Directed Mutagenesis kit (Stratagene, La Jolla, CA, USA). Transfection was performed using Effectene transfection reagent (Qiagen, Valencia, CA, USA).

Western blotting. HEK293T cells were lysed as previously described (3). Preparations of nuclear and cytoplasmic extracts were constructed using NucBuster Protein Extraction kit (Merck, Darmstad, Germany). Western blotting was performed as per standard protocols. The expression of proteins was examined using antibodies against cyclin D1 (ab16663; Abcam, Cambridge, UK), HA (\#2367; Cell Signaling Technology, Inc., Beverly, MA, USA) and p84 (ab487; Abcam). Antibody against $\beta$-actin (A2228; Sigma, St. Louis, MO, USA) was used as an internal control. The secondary antibodies used were NA931V and NV934V (GE Healthcare, Sydney, Australia). All antibodies were used at the recommended concentrations as directed by the manufacturer. The proteins were visualized using an ECL Western Blot Detection kit (Amersham Biosciences, Piscataway, NJ, USA).

Immunocytochemistry. Immunocytochemistry was performed as previously described (21). The primary antibodies used were the same as those for western blotting. The secondary antibodies were Alexa Fluor 488-conjugated donkey antimouse IgG and Alexa Fluor 555-conjugated goat anti-rabbit IgG (Invitrogen, Carlsbad, CA, USA). The primary and secondary antibodies were used at a 1:100 dilution. All slides were briefly counterstained with DAPI $(1 \mu \mathrm{g} / \mathrm{ml})$ for $5 \mathrm{~min}$ for nuclear determination and analyzed by confocal fluorescence microscopy (Carl-Zeiss MicroImaging Inc., Oberkochen, Germany).

Luciferase assays. HEK293T cells were transfected with wild-type (WT) cyclin D1 or T286I cyclin D1 expression plasmids $(0.4 \mu \mathrm{g})$ together with a reporter plasmid (pRb-TA-luc) $(0.25 \mu \mathrm{g})$. As an internal control, cells were co-transfected with phRL CMV-Renilla vector (Promega, Madison, WI, USA) (0.01 ng). Luciferase activity was detected using the Microplate Luminometer LB96V (EG\&G Breatholod, Wildbad, Germany). Each experiment was repeated three times.

Clonogenic cell survival assays. HEK293T cells were seeded in 6-well plates. After transfection with WT- or T286Icyclin D1, cells were incubated for 1 week with DMEM supplemented with G418 (1,000 $\mu \mathrm{g} / \mathrm{ml})$ (Invitrogen). The colonies were stained with Giemsa.

Statistical analysis. Statistical analyses of data from luciferase and clonogenic cell survival assays were carried out using the Student's t-test. $\mathrm{P}<0.05$ was considered to indicate a statistically significant result.

\section{Results}

T286I CCND1 mutation in endometrial cancer. Clinicopathological characteristics are examined and are presented in Table I. Briefly, sequencing of the CCNDl gene identified somatically acquired (non-germline) mutations in 2 out of 88 (2.3\%) endometrial cancer specimens (Fig. 1A). Both mutations were a substitution of amino acid 286 from threonine to isoleucine (T286I). These 2 patients died of the disease within 11 and 26 months of diagnosis, respectively. One case was stage IV endometrial carcinoma (grade 3), whereas the other was mixed adenocarcinoma with stage IIIc. Immunohistochemical staining of these 2 tumors showed accumulation of cyclin D1 in the nucleus (Fig. 1B). 
Table II. Clinicopathological background and mutation status of the two cases harboring the CCND1 mutation.

\begin{tabular}{lclccccccc} 
& & & & & & \multicolumn{4}{c}{ Mutation status } \\
\cline { 7 - 9 } $\begin{array}{l}\text { Case } \\
\text { no. }\end{array}$ & $\begin{array}{c}\text { Age } \\
\text { (years) }\end{array}$ & Histology & Grade & Stage & \begin{tabular}{c} 
Survival \\
\cline { 6 - 8 } (months)
\end{tabular} & CCNDI & K-Ras & PIK3CA & PTEN \\
\hline 1 & 52 & Endomerioid & 3 & 4 & 11 & T286I & G13D & H1047R & Del 135-141 (Del 19 bp, stop 140) \\
2 & 55 & Mixed & 1 & $3 \mathrm{c}$ & 26 & T286I & G12D & G1007R & R130G, Del 267 (Del A, stop 275) \\
\hline
\end{tabular}

A

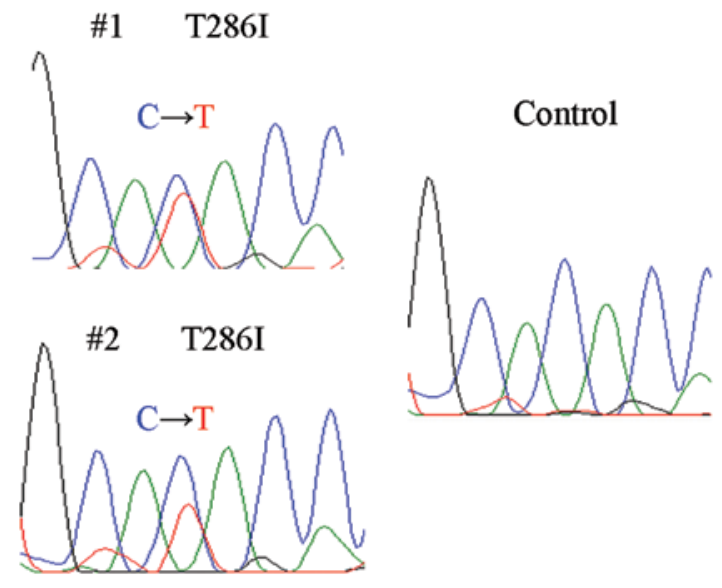

B

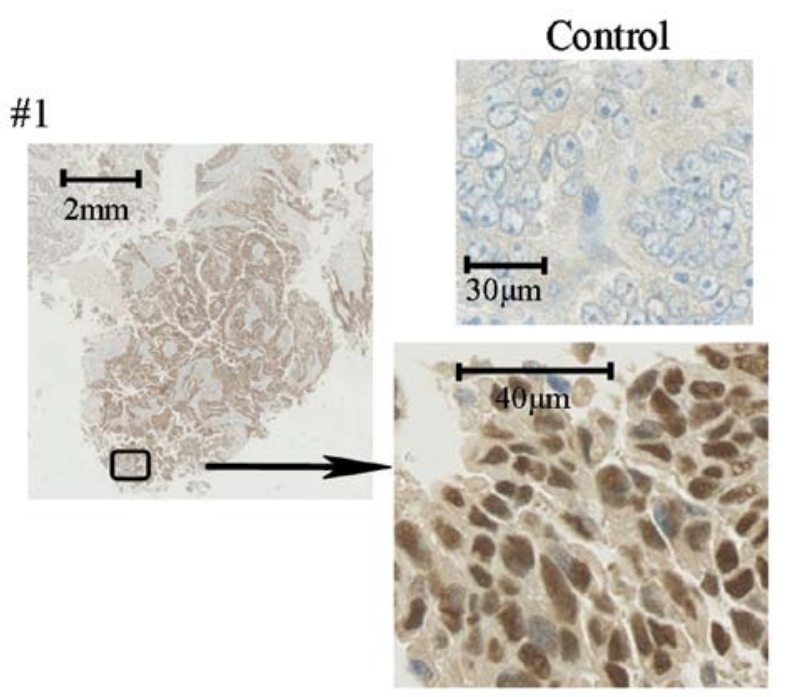

Figure 1. CCND1 mutations in 2 endometrial carcinomas and correlation with cyclin D1 nuclear accumulation. (A) PCR-direct sequence analysis of $C C N D 1$ in endometrial cancer tumors. Two of 88 samples (2.3\%) showed a single-base substitution in amino acid 286 from threonine to isoleucine. (B) Immunohistochemical analysis showed cyclin D1 expression in the nucleus in tumors harboring the cyclin D1-T286I mutation.

A

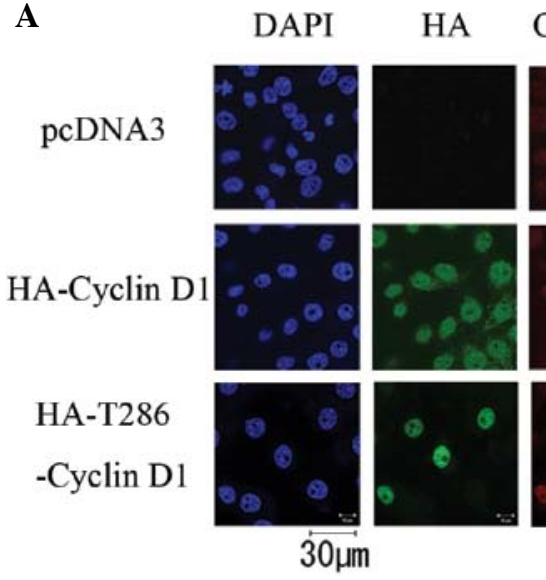

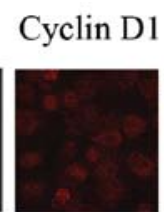

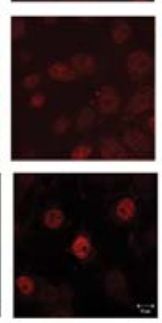

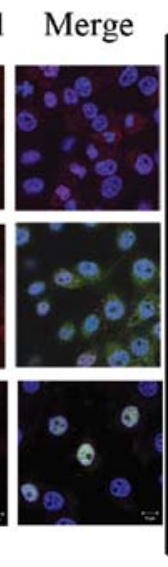

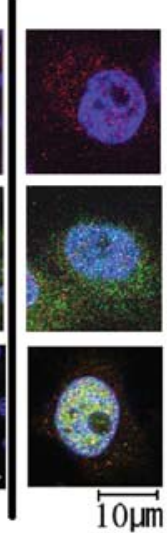

B

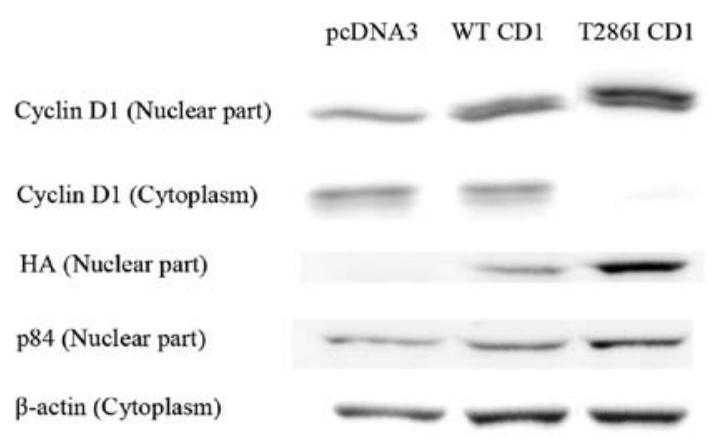

Figure 2. T286I mutation induces constitutive and stable nuclear cyclin D1 translocation in HEK293T cells. HEK293T cells were transfected with HA-tagged pcDNA3 (pcDNA3-CT) or HA-tagged WT cyclin D1 pcDNA3 (CD1-WT) or HA-tagged T286I cyclin D1 pcDNA3 (CD1-T286I). (A) Immunofluorescence showed cyclin D1 was overexpressed in the CD1-WT-expressing cells and was confined to the nucleus in the CD1-T286I-expressing cells. (B) Western blotting revealed that CD1-T286I transfection resulted in cyclin D1 stabilization in the nucleus and not in the cytoplasm.

CCND1 mutation at T286I coexists with triple mutations of $K$-Ras, PIK3CA and PTEN. Mutational analysis of $K$-Ras, $P I K 3 C A$ and PTEN was performed in both samples with the CCND1 mutation at T286I. Case no. 1 possessed overlapping mutations in K-Ras at G13D, in PIK3CA at H1047R and in PTEN (there was a deletion of 19 bp at codons 135-141). Case no. 2 also possessed these three mutations; in K-Ras at G12D, in PIK3CA at G1007R and in PTEN at R130G and deletion of 'A' in codon 267 (Table II).

T286I mutation induces constitutive nuclear cyclin DI accumulation. We introduced mutant cyclin D1 (T286I) into HEK293T cells, which do not possess CCND1 mutations. We transfected three types of pcDNA3 plasmids: pcDNA3 
A

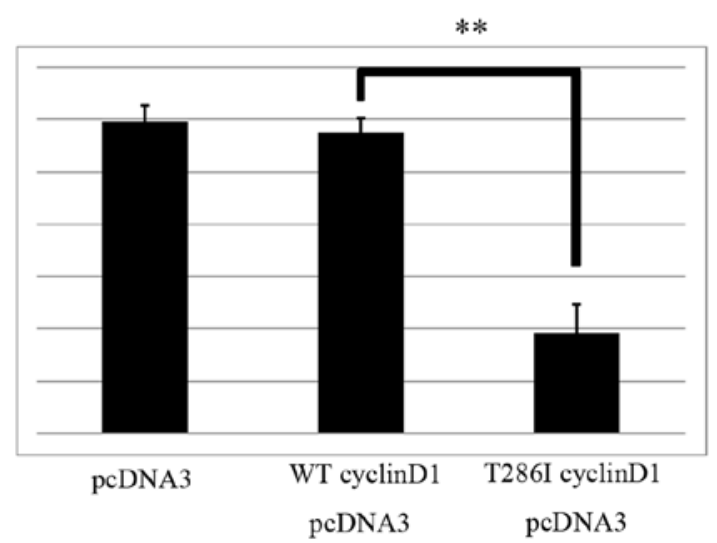

B

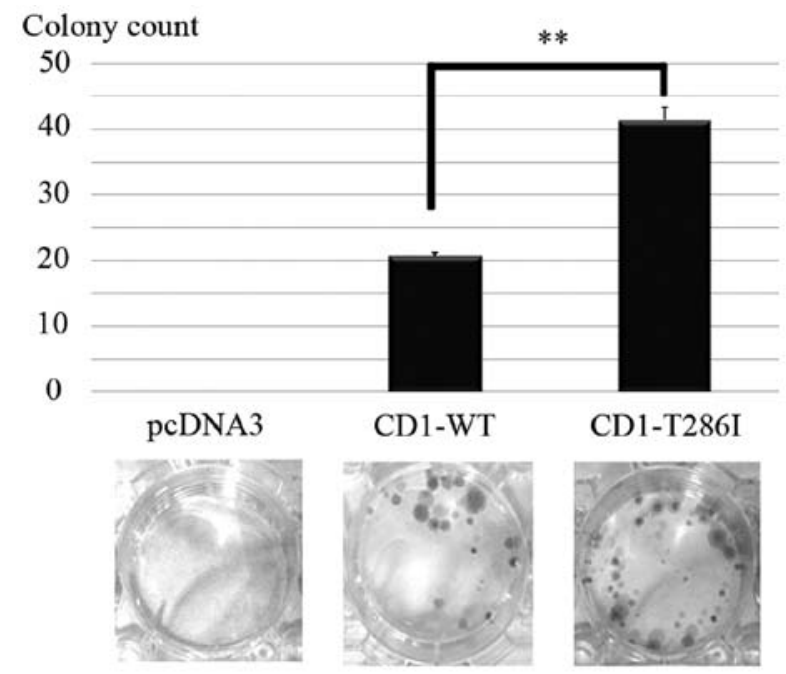

Figure 3. Cyclin D1-T286I mutation promotes oncogenic activation. (A) pRB transcriptional activity was examined in HEK293T cells transfected with CD1-WT, CD1-T286I or the control plasmid, by luciferase assays. CD1-T286I suppressed the expression of $\mathrm{pRb}$ protein. (B) Clonogenic cell survival assays were performed using similarly transfected HEK293T cells. After 1 week of selection in G418, colonies were stained with Giemsa and quantified. All experiments were repeated three times, and each value is shown as the mean of 3 experiments $\pm \mathrm{SD}\left({ }^{* *} \mathrm{P}<0.01\right)$. The result showed increased formation of colonies in cells with the CD1-T286I mutation.

as a control (pcDNA3-CT), HA-tagged WT cyclin D1 (CD1WT) and HA-tagged T286I mutant cyclin D1 (CD1-T286I). Immunocytochemistry indicated that endogenous cyclin D1 was localized to both the nucleus and cytoplasm in the pcDNA3CT-transfected cells (Fig. 2A). Exogenous CD1-WT was more clearly expressed in the nucleus than that in the cytoplasm. In the CD1-T286I-transfected cells, cyclin D1 was strongly accumulated in the nucleus, and endogenous cyclin D1 was undetectable in the cytoplasm. In western blot experiments, both nuclear and cytoplasmic expression of cyclin D1 was observed in the pcDNA3-CT- and CD1-WT-transfected cells (Fig. 2B). However, either endogenous or exogenous cyclin D1 was predominantly expressed in the nucleus in the D1-T286Itransfected cells. These results suggest that the CCNDI mutation at T286I prevents nuclear export of cyclin D1.

T286I mutation reduces $R b$ expression and promotes cell proliferation. As nuclear cyclin D1 and pRb expression are antagonistic for cell cycle progression, we transiently transfected cyclin D1 vectors and examined pRb transcriptional activity using luciferase assays. No significant difference was observed in $\mathrm{pRb}$ transcriptional activity by introduction of CD1-WT, when compared with pcDNA3-CT. However, CD1-T286I clearly suppressed the transcriptional activation of $\mathrm{pRb}(\mathrm{P}=0.002)$ (Fig. 3A).

Finally, to investigate the effects of T286I on cell proliferation, we performed clonogenic cell survival assays. No colonies were observed in the pcDNA3-CT-transfected cells, whereas colonies were observed in the CD1-WT cells (Fig. 3B). In addition, CD1-T286I further increased the number of colonies in HEK293T cells $(\mathrm{P}=0.007)$ (Fig. 3B). These results suggest that accumulation of mutant cyclin D1 (T286I) in the nucleus promotes cell cycle proliferation.

\section{Discussion}

Phosphorylation-dependent nuclear export of cyclin D1 at Thr286 is suggested to be critical for prevention of aberrant cell proliferation in vitro (22). This is the first report of $C C N D 1$ mutations at Thr286 in endometrial cancer. We identified this mutation (T286I) in two tumors (2.3\%) in association with accumulation of cyclin D1 protein in the nucleus.

Previously, Moreno-Bueno et al (19) reported the existence of CCND1 mutations at P287T, P287S and a $12 \mathrm{bp}$ in-frame deletion (289-292) in endometrial cancer. Benzeno et al (18) showed that these mutations specifically disrupt phosphorylation-dependent nuclear export of cyclin D1 and contribute to the genesis and progression of neoplastic growth. However, the function of the CCND1 mutation at T286I (CD1-T286I) has not been well characterized. Our results showed that CD1-T286I prevents the nuclear export of cyclin D1 and significantly promotes cell proliferation. These findings suggest that this CCNDI (T286I) mutation might promote tumor progression in certain endometrial carcinomas.

The functions of the CCNDI mutation in vivo were reported by Gladden et al (23). They showed that transgenic mice expressing mutant cyclin D1 (T286A) induced oncogenic nuclear retention of cyclin D1. This report is compatible with our data that a missense mutation at Thr286 disrupts nuclear export of cyclin D1. The clinical outcome of patients with cyclin D1 mutations were not reported in previous studies $(18,19)$. However, cyclin D1 overexpression is frequently observed in various types of cancers, and the prognostic impact of the overexpression was distinct among tumor types. Cyclin D1 overexpression is suggested to be induced by chromosomal translocation, gene amplification and protein stabilization $(24,25)$. The prognosis of patients with cyclin D1 overexpression is poor in ovarian cancer (26) and favorable in colorectal cancer (27). These contradictory results may be caused by additional genetic alterations in each cancer type. Indeed, expression of wild-type cyclin D1 in murine fibroblasts or lymphocytes does not promote neoplastic transformation without co-expression of a cooperating oncogene such as Myc or Ras (28-31). In the present study, both patients with mutant cyclin D1 (T286I) had poor prognosis as they harbored coexisting triple mutations of K-Ras, PTEN and $P I K 3 C A$. Although the sample size was extremely small, our data may suggest that activation of both Ras/MAPK and PI3K 
pathways cooperate with mutant cyclin D1 and promote tumor aggressiveness in certain endometrial carcinomas. Further study is warranted to clarify the significance of CCNDI mutations in endometrial cancer.

\section{Acknowledgements}

We thank Keiko Shoji, Yuichiro Miyamoto, Yoko Matsumoto, Takahide Arimoto, Kensuke Tomio, Satoko Kojima and Reiko Kurikawa for their support and assistance.

\section{References}

1. Jemal A, Siegel R, Xu J and Ward E: Cancer statistics, 2010. CA Cancer J Clin 60: 277-300, 2010.

2. McMeekin DS, Filiaci VL, Thigpen JT, Gallion HH, Fleming GF and Rodgers WH: The relationship between histology and outcome in advanced and recurrent endometrial cancer patients participating in first-line chemotherapy trials: a Gynecologic Oncology Group study. Gynecol Oncol 106: 16-22, 2007.

3. Oda K, Stokoe D, Taketani Y and McCormick F: High frequency of coexistent mutations of PIK3CA and PTEN genes in endometrial carcinoma. Cancer Res 65: 10669-10673, 2005.

4. Oda K, Okada J, Timmerman L, et al: PIK3CA cooperates with other phosphatidylinositol 3'-kinase pathway mutations to effect oncogenic transformation. Cancer Res 68: 8127-8136, 2008.

5. Shoji K, Oda K, Nakagawa $\mathrm{S}$, et al: The oncogenic mutation in the pleckstrin homology domain of AKT1 in endometrial carcinomas. Br J Cancer 101: 145-148, 2009.

6. Murayama-Hosokawa S, Oda K, Nakagawa S, et al: Genomewide single-nucleotide polymorphism arrays in endometrial carcinomas associate extensive chromosomal instability with poor prognosis and unveil frequent chromosomal imbalances involved in the PI3-kinase pathway. Oncogene 29: 1897-1908, 2010.

7. Ikeda Y, Oda K, Nakagawa S, et al: Genome-wide single nucleotide polymorphism arrays as a diagnostic tool in patients with synchronous endometrial and ovarian cancer. Int J Gynecol Cancer 22: 725-731, 2012.

8. Malumbres M and Barbacid M: Cell cycle, CDKs and cancer: a changing paradigm. Nat Rev Cancer 9: 153-166, 2009.

9. Kato J, Matsushime H, Hiebert SW, Ewen ME and Sherr CJ Direct binding of cyclin $\mathrm{D}$ to the retinoblastoma gene product (pRb) and pRb phosphorylation by the cyclin D-dependent kinase CDK4. Genes Dev 7: 331-342, 1993.

10. Lundberg AS and Weinberg RA: Functional inactivation of the retinoblastoma protein requires sequential modification by at least two distinct cyclin-cdk complexes. Mol Cell Biol 18: 753-761, 1998.

11. Tetsu $\mathrm{O}$ and McCormick $\mathrm{F}$ : $\beta$-Catenin regulates expression of cyclin D1 in colon carcinoma cells. Nature 398: 422-426, 1999.

12. Albanese $\mathrm{C}$, Johnson J, Watanabe G, et al: Transforming p21ras mutants and c-Ets-2 activate the cyclin D1 promoter through distinguishable regions. J Biol Chem 270: 23589-23597, 1995.

13. Musgrove EA, Caldon CE, Barraclough J, Stone A and Sutherland RL: Cyclin D as a therapeutic target in cancer. Nat Rev Cancer 11: 558-572, 2011.
14. Knudsen KE, Diehl JA, Haiman CA and Knudsen ES: Cyclin D1: polymorphism, aberrant splicing and cancer risk. Oncogene 25: 1620-1628, 2006.

15. Solomon DA, Wang Y, Fox SR, et al: Cyclin D1 splice variants. Differential effects on localization, RB phosphorylation, and cellular transformation. J Biol Chem 278: 30339-30347, 2003.

16. Lu F, Gladden AB and Diehl JA: An alternatively spliced cyclin $\mathrm{D} 1$ isoform, cyclin D1b, is a nuclear oncogene. Cancer Res 63: 7056-7061, 2003

17. Kang S, Kim JW, Park NH, Song YS, Kang SB and Lee HP: Cyclin D1 polymorphism and the risk of endometrial cancer. Gynecol Oncol 97: 431-435, 2005.

18. Benzeno S, Lu F, Guo M, et al: Identification of mutations that disrupt phosphorylation-dependent nuclear export of cyclin D1. Oncogene 25: 6291-6303, 2006.

19. Moreno-Bueno G, Rodriguez-Perales S, Sanchez-Estevez C, et al: Cyclin D1 gene (CCND1) mutations in endometrial cancer. Oncogene 22: 6115-6118, 2003.

20. Minaguchi T, Yoshikawa $\mathrm{H}$, Oda $\mathrm{K}$, et al: PTEN mutation located only outside exons 5, 6, and 7 is an independent predictor of favorable survival in endometrial carcinomas. Clin Cancer Res 7: 2636-2642, 2001

21. Tanikawa M,Wada-Hiraike O,Nakagawa S, et al: Multifunctional transcription factor TFII-I is an activator of BRCA1 function. $\mathrm{Br}$ J Cancer 104: 1349-1355, 2011.

22. Alt JR, Cleveland JL, Hannink M and Diehl JA: Phosphorylation-dependent regulation of cyclin D1 nuclear export and cyclin D1-dependent cellular transformation. Genes Dev 14: 3102-3114, 2000.

23. Gladden AB, Woolery R, Aggarwal P, Wasik MA and Diehl JA: Expression of constitutively nuclear cyclin D1 in murine lymphocytes induces B-cell lymphoma. Oncogene 25: 998-1007, 2006.

24. Hall $\mathrm{M}$ and Peters G: Genetic alterations of cyclins, cyclindependent kinases, and Cdk inhibitors in human cancer. Adv Cancer Res 68: 67-108, 1996.

25. Sherr CJ: Cancer cell cycles. Science 274: 1672-1677, 1996.

26. Bali A, O'Brien PM, Edwards LS, Sutherland RL, Hacker NF and Henshall SM: Cyclin D1, p53, and p21 $1^{\text {Waf1/Cip1 }}$ expression is predictive of poor clinical outcome in serous epithelial ovarian cancer. Clin Cancer Res 10: 5168-5177, 2004

27. Ogino S, Nosho K, Irahara N, et al: A cohort study of cyclin D1 expression and prognosis in 602 colon cancer cases. Clin Cancer Res 15: 4431-4438, 2009.

28. Bodrug SE, Warner BJ, Bath ML, Lindeman GJ, Harris AW and Adams JM: Cyclin D1 transgene impedes lymphocyte maturation and collaborates in lymphomagenesis with the myc gene. EMBO J 13: 2124-2130, 1994.

29. Lovec H, Grzeschiczek A, Kowalski MB and Moroy T: Cyclin D1/bcl-1 cooperates with myc genes in the generation of B-cell lymphoma in transgenic mice. EMBO J 13: 3487-3495, 1994.

30. Lovec H, Sewing A, Lucibello FC, Muller R and Moroy T: Oncogenic activity of cyclin D1 revealed through cooperation with Ha-ras: link between cell cycle control and malignant transformation. Oncogene 9: 323-326, 1994.

31. Uchimaru K, Endo K, Fujinuma H, Zukerberg L, Arnold A and Motokura T: Oncogenic collaboration of the cyclin D1 (PRAD1, bcl-1) gene with a mutated p53 and an activated ras oncogene in neoplastic transformation. Jpn J Cancer Res 87: 459-465, 1996. 